\title{
Energías renovables y sustentabilidad: una eficiente forma de gestionar los recursos naturales
}

\author{
Ernesto Jesús Perino, Roberto Aníbal Kiessling Duran, \\ Adolfo Alejandro Silnik, Aníbal Daniel Perelló y Ernesto Perino
}

\begin{abstract}
Resumen
Este trabajo aborda la crisis socioecológica actual ocasionada por el cambio climático, y centra su análisis en la promoción de alternativas para enfrentarla; también, se resalta el papel de la ética ambiental, campo del saber que focaliza sus reflexiones en la relación del hombre con el medioambiente. De igual manera, este artículo, demanda un punto de equilibrio entre la ciencia, el desarrollo tecnológico y el rol de las energías renovables para alcanzar una biosfera sostenible. Es importante considerar que este paradigma emergente instala la necesidad de reconocer los derechos intrínsecos de la esfera de la vida y vislumbra la conjunción indisoluble: energías renovables-sostenibilidad como un objetivo válido para el bienestar del medioambiente.
\end{abstract}

Palabras clave: energías renovables, sostenibilidad, ética ambiental, medioambiente.

\section{RENEWABLE ENERGIES AND SUSTAINABILITY: AN EFFICIENT WAY TO MANAGE NATU-}

\section{RAL RESOURCES}

\begin{abstract}
This paper addresses the current socio-ecological crisis. It focuses its analysis on a promotion of alternatives for dealing with the global crisis and climate change. It is worth highlighting the role of environmental ethics; a field of knowledge that focuses its reflections on the relationship between man and the environment. It demands a point of balance between science, technological development and the role of renewable energies to achieve a sustainable biosphere. This emerging paradigm installs the need to recognize those of rights intrinsic to the sphere of life. The indissoluble conjunction renewable energies- sustainability is seen as a valid objective for the environmental welfare.
\end{abstract}

Keywords: renewable energies, sustainability, environmental ethics, environment. 
"Energías renovables y sustentabilidad: una eficiente forma de gestionar los recursos naturales" Ernesto Jesús Perino, Roberto Aníbal Kiessling Duran, Adolfo Alejandro Silnik, Aníbal Daniel Perelló y Ernesto Perino Vol. 22, Núm. 3, mayo-junio 2021 Revista Digital Universitaria

\section{Ernesto Jesús Perino}

$$
\begin{array}{r}
\text { ejperino@unsl.edu.ar } \\
\text { orcid.org/0000-0001-9137-3838 }
\end{array}
$$

Ingeniero Electrónico por parte de la Universidad Nacional de San Luis (UNSL), Argentina. Realizó un Máster en la Universidad de Cádiz (UCA), España, en Gestión de Energías Renovables. Experto Universitario en Diseño de Plantas Fotovoltaicas por la Universidad Tecnológica Nacional, Facultad Regional Buenos Aires (UTN-FRBA), Argentina. Docente e investigador del departamento de física y del departamento de electrónica de la unsL. Integrante de diversos proyectos y comisiones en la unsL, tales como el Proyecto Radiación Solar y Medio Ambiente, la comisión de carrera Tecnicatura Universitaria en Energías Renovables, y la comisión evaluadora de la creación de Ingeniería en Energías Renovables.

\section{Roberto Aníbal Kiessling Duran}

rkiessling@unsl.edu.ar orcid.org/0000-0002-9402-397X

Ingeniero en Electrónica y Electricidad por parte de la Facultad de Ingeniería, Universidad de Mendoza. Realizó un máster en II Facoltá di Ingeniería, Politecnico di Torino en Wireless Systems and Related Technologies. Docente e investigador del departamento de electrónica perteneciente a la Facultad de Ciencias Físico Matemáticas y Naturales de la Universidad Nacional de San Luis (unsL) e integrante del Proyecto Desarrollo de Sistemas Embebidos Interconectados para Aplicaciones AgroTics, en la unsL.

\section{Adolfo Alejandro Silnik}

aasilnik@unsl.edu.ar

orcid.org/0000-0003-4413-5233

Ingeniero en Electrónica por parte de la Universidad Tecnológica Nacional, Facultad Regional Córdoba, Argentina. Especialista en Redes de Datos por la Universidad Tecnológica Nacional, Facultad Regional Mendoza, Argentina. Docente e investigador del departamento de electrónica de la Universidad Nacional de San Luis (UNSL), Argentina. Integrante del Proyecto Desarrollo de Sistemas Embebidos Interconectados para Aplicaciones AgroTics, UNSL.

\section{Aníbal Daniel Perelló}

danielperello958@gmail.com orcid.org/0000-0001-7336-9894

Licenciado en Física por parte de la Universidad Nacional de San Luis (UNSL), Argentina. Magíster en Energías Renovables, Universidad Nacional de Salta (unsa), Argentina. Profesor adjunto exclusivo e investigador del departamento de física de la unsL y profesor asociado de la Universidad Nacional de Villa Mercedes (unvime), Argentina. Es integrante del Proyecto radiación solar y medio ambiente, de la unsL. Director de carrera de la Tecnicatura Universitaria en Energías Renovables, unsL. Es integrante de dos comisiones dentro de la unsL: comisión de carrera Tecnicatura Universitaria en Energías Renovables, unsL y de la comisión evaluadora de la creación de Ingeniería en Energías Renovables, unsL.

\section{Ernesto Perino}

perinoernesto@gmail.com orcid.org/0000-0001-5244-7312

Licenciado en Ciencias Geológicas por parte de la Universidad Nacional de San Luis (UNSL), Argentina. Realizó un doctorado en la UNsL en Química con la tesis titulada Desarrollo de Técnicas Analíticas por FRX aplicadas a la Prospección Geoquímica de Pegmatitas Graníticas. Profesor titular exclusivo e investigador de la Facultad de Química Bioquímica y Farmacia (UNSL). Es integrante de diversos proyectos y comisiones, tales como el Proyecto Desarrollo y Aplicación de Metodologías Analíticas destinadas a la Determinación y Especiación Analitos Orgánicos e Inorgánicos en Muestras de Interés Biológico, Farmacéutico, Tecnológico, Ambiental y Alimentario; del Consejo de Área de Química Analítica del departamento de química de la FQByF (UNSL) y en esa misma institución, forma parte del Consejo de Admisión de la Maestría en Química Analítica del departamento de química. 


\section{Introducción}

El progreso científico y tecnológico, en la sociedad, le brinda al hombre la posibilidad de ejercer un gran poder sobre su entorno, en consecuencia, esto ocasiona diversas problemáticas ambientales que ponen en peligro a la biosfera, fracción del planeta en la que se desarrolla la vida y que integra a todos los ecosistemas ${ }^{1}$, ya sean terrestres, acuáticos, aéreos o de transición. El ser humano actúa descontroladamente (por ejemplo, la ignición de combustibles fósiles, la deforestación, etc.), lo cual genera un colapso medioambiental, motivo de gran preocupación para la ética ambientalista, ya que este campo del saber emerge como bastión para indicarle a la humanidad que debe recuperar la consciencia de que vive en un planeta sagrado del cual forma parte.

Frente a los planteamientos anteriores, este trabajo apunta a concientizar sobre la necesidad de un manejo equilibrado de los recursos naturales, y estimula un compromiso con la transición hacia los sistemas energéticos que tengan una amplia participación de las energías renovables y que generen una huella de carbono neutral ${ }^{2}$ a partir de la implementación del concepto de las cuatro Rs del desarrollo sostenible: Reducir, Reutilizar, Reciclar y Reeducar. Estos cambios conductuales demandan privilegiar las visiones ambientalistas y resaltan la necesidad de situar a las instituciones educativas dentro de un marco ambiental, ya que son escenarios vitales para promover la sustentabilidad. El paradigma de la sostenibilidad se encuentra también en la práctica universitaria en una dimensión educativa, investigativa y de transferencia social (ver imagen 1).

Imagen 1. Reparación y reutilización de módulos Fv donados al LEIS - UNSL. Esta tarea apunta a la reeducación sustentable en el ámbito universitario. Fuente: fotografía propia.

1 Sistema funcional formado por el medio físico y la comunidad que lo habita.

2 Equilibrio entre la cantidad de dióxido de carbono equivalente liberado a la atmósfera con una cantidad simétrica retirada de ella.

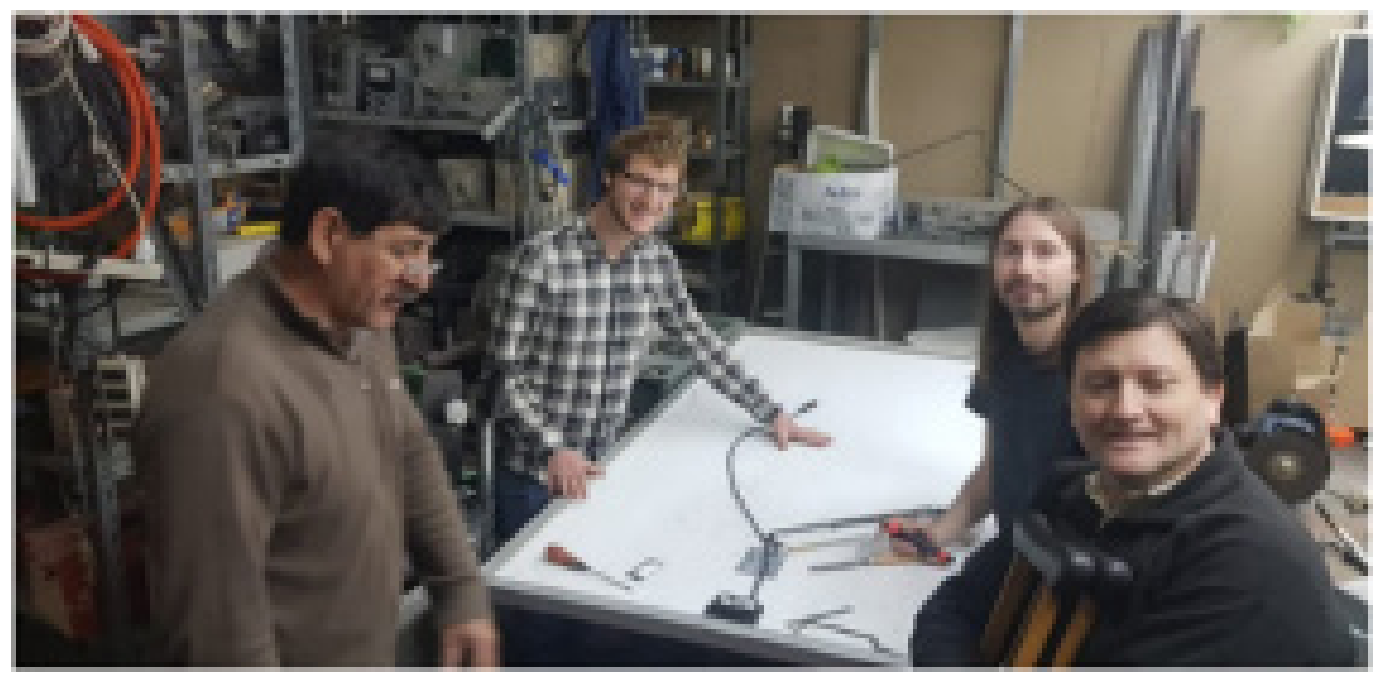

Jacques Lacan, en 1953, pedía a los intelectuales y políticos: "... que renuncie quien no pueda unir a su horizonte la subjetividad de su época" (2009, p. 308). En un momento donde el planeta enfrenta serias amenazas que ponen en riesgo la vida sobre la tierra, no cabe duda de que el medioambiente es una marca epocal subjetiva, en otras palabras, nuestro presente lleva las marcas significativas de la contaminación ambiental generada a gran escala y a un ritmo exacerbado en los 
últimos años, lo cual le impide a la biosfera autorregularse, es decir, no puede tener la capacidad de controlar globalmente el ambiente para amoldarlo a sus propias necesidades (Hipótesis de Gaia).

Este panorama sombrío exige una metamorfosis profunda y, en muchos casos, contradictoria al actual modo de vida. Antes de que colapse la biosfera por la acción antropogénica ${ }^{3}$, es necesario abordar la problemática ambiental mediante un análisis profundo de la relación hombre-sociedad-naturaleza, lo que implica la adopción de un novedoso modelo integral, asentado en los Objetivos del Desarrollo Sostenible (oDs) que demandan la evolución armoniosa de los ecosistemas sin exceder sus límites de capacidad de carga.

Los principios rectores de estos Objetivos son la equidad social y la preservación de la biosfera, lo cual implica que los procesos antrópicos sean armónicos ya que pretenden alcanzar "una visión holística de la naturaleza asociada a la recuperación de una vida saludable, verdadera e íntegra" (Agoglia Moreno, 2010, p. 87). Esta realidad justifica el porqué la cuestión ambiental ha revolucionado la mirada con la vemos el mundo, y nos obliga a plantear nuevos paradigmas y a tener una actitud integradora en el abordaje de las prácticas científicas y tecnológicas ecoamigables, junto con otras modalidades de construcción en la interacción sociedad-naturaleza.

\section{Ventana de oportunidades tras la sostenibilidad}

Como puntualiza Kalmus: "La civilización está en una encrucijada. A escala global, los seres humanos son ahora la principal perturbación para el mundo natural [...] Las dos crisis que debe afrontar con más urgencia la biosfera, y con ella la humanidad, son el cambio climático y la pérdida de hábitats" (2018, p. 222). Estas razones exigen un cambio socio-cultural paradigmático; nuevos modos de ser y de saber; un giro en nuestra concepción antropocéntrica; un repensar como antrophos, para generar un puente entre lo antrópico y lo no antrópico, y poner en marcha una revolución que considere como pilares de un desarrollo sustentable a los recursos renovables. Una revolución inaugurada en el nuevo milenio y que pueda caracterizarse por la diseminación de las energías renovables y de las nuevas tecnologías; que pueda extender la sensibilidad empática a toda la vida terrenal (Rifkin, 2010).

La necesidad de buscar fórmulas más sostenibles para interactuar con el entorno coloca a la tecnociencia ${ }^{4}$ en un escenario central. Desde este ángulo, se puede afirmar que jla hora de las energías alternativas y amigables con el

${ }^{3}$ Las acciones antropológicas son las transformaciones ejercidas por el hombre sobre la naturaleza.

${ }^{4}$ Instrumentalización tecnológica del conocimiento científico. medioambiente ha llegado! Las tecnologías de energías renovables son un elemento vital y unas alternativas técnicas y económicamente viables. En la actualidad, el papel del sistema energético constituye una de las prioridades de la agenda científica, política, económica y social debido a las amplias repercusiones que tiene este sistema sobre el conjunto del planeta. 
En este sentido, se han generado nuevas estrategias, y se ha invertido en numerosos recursos para desarrollar y consolidar un modelo energético con un enfoque centrado en valores con prioridades ecológicas que apunten al bien común para encontrar soluciones que afronten los desafíos futuros. Este modelo se sustenta en la variedad de opciones disponibles que ofrece la naturaleza para el aprovechamiento de energías limpias y primordialmente, trata de saber cómo convertir la radiación solar, el viento, la biomasa o el agua en electricidad, calor o energía de la manera más eficiente, sostenible y económicamente posible. Así, una matriz energética basada en fuentes de energías renovables (solar, eólica, biomasa, geotérmica, hidráulica, etc.) tiene diferentes consecuencias sobre un sinnúmero de cuestiones de carácter ético-ecológico para el análisis de estrategias en el marco del desarrollo sostenible.

Se vislumbra que las tecnologías de energías renovables, principalmente los sistemas eólicos y solares fotovoltaicos, son opciones prometedoras para un futuro de carbono neutro. Por tanto, el cuidado de los recursos naturales es un imperativo categórico de la humanidad. El desarrollo sustentable se puede personificar mediante la triada equilibrada de sociedad, medioambiente y economía (Perino et al., 2019; ver imagen 2).

Imagen 2. Triada del desarrollo sustentable. Fuente: elaboración propia.

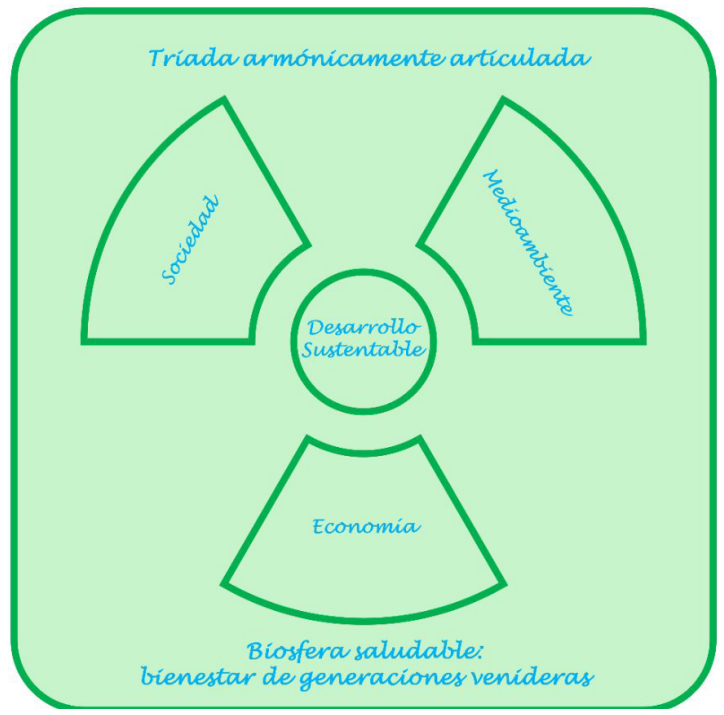

Bajo estos puntos, responder al imperativo ético-ecológico de la época implica basarse en el convencimiento de que la única vía para tratar las cuestiones ambientales, a partir de soluciones a escala global, es mediante la implementación de fuentes energéticas renovables y de la eficiencia energética; una ventana de oportunidades tras la sostenibilidad de la Tierra. Para avanzar en esta dirección, se requiere de un consenso solidario interregional e intergeneracional; de la superación de la crisis epistémica mediante una perspectiva biocéntrica que privilegie el valor moral de todo ser vivo; y de alcanzar una mayor integración entre las formas de vida y el desarrollo tecnocientífico con los intereses ambientales y socioculturales. 
"Energías renovables y sustentabilidad: una eficiente forma de gestionar los recursos naturales" Ernesto Jesús Perino, Roberto Aníbal Kiessling Duran, Adolfo Alejandro Silnik, Aníbal Daniel Perelló y Ernesto Perino

Vol. 22, Núm. 3, mayo-junio 2021

Revista Digital Universitaria

La investigación y el desarrollo de tecnologías para el aprovechamiento de fuentes de energías renovables presentan particularidades tecnocientíficas, ya que contribuyen al restablecimiento de las condiciones necesarias para alcanzar el equilibrio entre los procesos antrópicos y a la capacidad regenerativa de la biosfera. Es decir, se estaría en presencia de una eficiente forma de gestionar los recursos de la naturaleza que vela por el bienestar de las generaciones futuras y del ecosistema global.

Para Leff,

La crisis ambiental de nuestro tiempo es el signo de una nueva era histórica. Esta encrucijada civilizatoria es ante todo una crisis de la racionalidad de la modernidad y remite a un problema del conocimiento. La degradación ambiental -la muerte entrópica del planeta - es resultado de las formas de conocimiento a través de las cuales la humanidad ha construido el mundo y lo ha destruido por su pretensión de unidad, de universalidad, de generalidad y de totalidad; por su objetivación y cosificación del mundo (2007, p. 11).

Con esto, el autor invita a recapacitar en la necesidad de crear un nuevo marco conceptual para pensar la tecnociencia y para enfrentar sus desafíos. En este universo, los nuevos presupuestos epistémicos deben poner el acento en el hombre, en sus necesidades y en su interrelación con la naturaleza. Lo ambiental no es ajeno a la crisis civilizatoria de nuestro tiempo ya que presenta una excelente oportunidad para pensar en alternativas radicales. El viraje hacia un paradigma ecológico se debe dar en la ciencia, en los valores y en las actitudes individuales y colectivas, así como en los nuevos patrones de desarrollo socioeconómicos. En resumen, el avance tecnocientífico constituye un instrumento esencial para la concreción de un profundo reajuste sociocultural, económico y medioambiental.

Resulta oportuno destacar las ideas del escritor Ernesto Sábato al considerar la crisis de la sociedad contemporánea:

Asistimos a una quiebra total de la cultura occidental. El mundo cruje y amenaza con derrumbarse, ese mundo que para mayor ironía es el resultado de la voluntad del hombre, de su prometeico intento de dominación. A cada hora el poder del mundo se concentra y se globaliza. Veinte o treinta empresas, como un salvaje animal totalitario, lo tienen en sus garras. Continentes en la miseria junto a altos niveles tecnológicos, posibilidades de vida asombrosas a la par de millones de hombres desocupados, sin hogar, sin asistencia médica, sin educación. (...) Esta crisis no es la crisis del sistema capitalista, como muchos imaginan: es la crisis de toda una concepción del mundo y de la vida basada en la idolatría de la técnica y en la explotación del hombre (2009, p. 56).

En otras palabras, el hombre ha perdido el sentido de los valores éticos mientras que el mundo global atenta contra la redistribución equitativa y responsable de los recursos naturales. Ambos problemas van en contra de los Objetivos del desarrollo sustentable. 
En esta instancia, parece pertinente recapacitar de dónde se viene y a dónde se va. Esto exige la necesidad de establecer las bases de un aparato ideológico político que priorice un medioambiente de calidad, y una nueva manera de comprender y de construir los sistemas socio-culturales. El contexto actual de la crisis socio-ecológica planetaria pone en evidencia la necesidad de políticas públicas verdes orientadas a la mitigación del cambio climático y a la conservación de la biodiversidad, como el fomento de las energías renovables, la reducción de emisiones contaminantes, la asequibilidad del agua potable, planes de manejo de desechos, programas de pesca sustentable, salud y educación ambiental, entre otras. Con lo anterior, se puede visualizar que el desarrollo tecnocientífico, las emergentes posibilidades de implementación de energías renovables y el uso racional y eficiente de la energía abren otras instancias de intervención sobre la biosfera, y promueven un desafío a la reflexión ética medioambiental. Dentro de este espíritu reflexivo, sujeto y naturaleza deben conformar una concepción holística: dualidad hombre-naturaleza.

¡La conciencia ecológica exige una actitud diferente! Obliga al hombre a reexaminar sus valores, a peregrinar hacia una construcción ética ambiental que concibe al planeta como la esfera de la vida en la que se encuentra inmersa la especie humana, y en consecuencia, a plantearse fuertes interrogantes: ¿Qué características y cómo se puede construir un mundo que restablezca la armonía entre los seres humanos y la naturaleza? ¿Cuáles deben ser los principios fundamentales de ese otro mundo necesario? ¿Cuál debe ser la responsabilidad de la tecnociencia ante el trinomio sociedad, medioambiente y economía? ¿Cuáles son los criterios para la elección de determinadas tecnologías y fuentes de energías renovables? ¿Cuáles son los impactos ambientales y qué resoluciones se pueden adoptar para reducir dichos impactos? Frente a estos cuestionamientos, se observa que el vertiginoso progreso tecnocientífico, vinculado a las energías renovables, ocupa un posicionamiento estratégico medioambiental.

Una de las opciones más prometedoras en la actualidad para contribuir a un marco energético sostenible, dentro de las diferentes fuentes energéticas, es la basada en la conversión fotovoltaica, debido a que, además de producir energía eléctrica de una fuente inagotable, obtenida directamente a partir de la radiación solar, el desarrollo de la energía solar fotovoltaica está teniendo un acelerado avance tecnológico y económico. Además, la generación de energía eléctrica directa obtenida de la radiación solar mediante sistemas fotovoltaicos no requiere de ningún tipo de combustión, por lo que no se produce polución térmica ni emisiones de dióxido de carbono (principal contribuyente al efecto invernadero antrópico). La energía solar fotovoltaica es la tercera fuente de energía renovable más importante, en términos de capacidad instalada a nivel mundial, tras la hidroeléctrica y la eólica.

Las energías renovables constituyen una fuente inagotable frente a los combustibles fósiles; promueven el autoabastecimiento de la matriz energética de un país; y son menos perjudiciales para el medioambiente, debido a que 
"Energías renovables y sustentabilidad: una eficiente forma de gestionar los recursos naturales" Ernesto Jesús Perino, Roberto Aníbal Kiessling Duran, Adolfo Alejandro Silnik, Aníbal Daniel Perelló y Ernesto Perino

Vol. 22, Núm. 3, mayo-junio 2021

Revista Digital Universitaria

contribuyen a la mitigación del cambio climático. De ello se desprende que, la sustentabilidad y la compleja imbricación de la energía constituyen la piedra angular frente a un proceso de cambio dirigido a la configuración de la sociedad, un proceso donde son igual de importantes tanto las metas globales como los caminos alternativos para arribar a ellas. La crisis medioambiental no se va a afrontar al menos que se alcance un acuerdo revelador y significante entre la naturaleza y el hombre, mientras se trate de invertir los efectos provocados por la contaminación y el calentamiento global antropogénico desmesurado. La gravedad de la temática pone a la sociedad frente a un agente movilizador a nivel mundial y abre las puertas a otras formas de valoración ambiental y cultural.

Una vida humana en armonía con la naturaleza, que también "posee derechos", entraña un tipo de vida inspirada en valores ético ambientales, diferentes a los del modelo de desarrollo imperante (Lecaros Urzúa, 2013). En esta directriz, Potter (1971) sostiene que la humanidad requiere, de manera imperiosa, una nueva sabiduría que le brinde la comprensión de cómo usar el conocimiento para la supervivencia y la mejoría de la humanidad. Delgado Díaz, considera que el precepto de la bioética como un nuevo campo del saber reposiciona al conocimiento científico a través de una nueva escala valores, y argumenta: "Es sumamente significativo que la propuesta de un nuevo saber desde la práctica de la vida - la bioética holística de Potter - coincidiera en su versión definitiva con las ideas elaboradas por el ambientalismo, y se proyectase así, como una ética ambiental, ecológica" (2002, p. 153). De ahí que, un saber ambiental junto con una intervención tecnológica con ética y con un planteamiento epistemológico donde se reafirmen los valores de la relación sociedad-naturaleza, conlleve al desafío de pensar alternativas y de elaborar estrategias transformadoras.

En este universo donde está inmersa la sociedad del conocimiento, caracterizada por la importancia que adquiere el saber en todos los ámbitos de la vida humana, la sobrevivencia del hombre en el planeta dependerá de la manera en que se asuma la nueva condición contemporánea del Saber (Osorio García, 2016). Por lo que, sería difícil comprender la complejidad del mundo circundante sin entender el papel que la ciencia y la tecnología cumplen dentro de la cultura moderna. Reflexionar desde esta complejidad implica pensar en procesos dinámicos y asumir una actitud reflexiva y crítica ante esta realidad. La ciencia, concebida como una actividad transformadora del mundo, debe asumir como suyos los retos que presentan la propia esencia del hombre y su hábitat natural y social.

Es a partir de este supuesto que cobra importancia la construcción de un sentido ético de la vida, un sentido moralizador que permita conciliar las necesidades de la protección medioambiental junto con las del hombre. Surge así un nuevo horizonte interpretativo... Frente a la urgencia de cambios con una profunda raíz ecológica, debido al progresivo colapso ocasionado por el cambio climático y la nocividad de los combustibles fósiles, el consumo de energía y las diferentes fuentes energéticas tienen una fuerte implicancia sobre numerosas cuestiones de carácter ético. 
A lo largo de la historia, la disponibilidad y asequibilidad de la energía se han convertido en un motor de progreso y de bienestar social. Desde una perspectiva actual, volcar la mirada hacia un modelo energético sustentable es, evidentemente, el nuevo imperativo categórico para salvar al planeta y el paso más inteligente que se puede dar para dejar un mundo mejor a las futuras generaciones. Se vuelve necesaria una ordenación racional y equitativa de los intereses comunes de la humanidad. La crisis axiológica conduce, inevitablemente, a la construcción de un proyecto humanista, ajustado a las condiciones de la sociedad contemporánea, donde impere la capacidad de empatía, de solidaridad y la voluntad de un trabajo colectivo.

La protección del medio ambiente, como uno de los ultimátums más destacado que ha enfrentado la humanidad, debe representar un firme compromiso interregional e intergeneracional encaminado al resguardo de nuestro entorno; porque es, ante todo, la sociedad quien debe reclamar una ética medioambiental. El despliegue de las energías renovables demuestra que el camino hacia un modelo energético sostenible no es un problema meramente técnico o económico, sino un problema sociocultural que es necesario abordar en conjunto; de tal manera que, los responsables de tomar las decisiones energéticas lo hagan desde una visión sustentable con el fin de preservar los factores bióticos, abióticos y socioeconómicos. ¡La existencia es una lucha por la energía ecosustentable! Un modelo energético como el que se busca sólo será concebible dentro de un proceso de transición global hacia las fuentes de energías renovables, en una sociedad diferente, apoyada en valores diferentes (ver imagen 3).

Imagen 3. Energías renovables y desarrollo sustentable. Fuente: Arq. Nicolás A. Bontá.

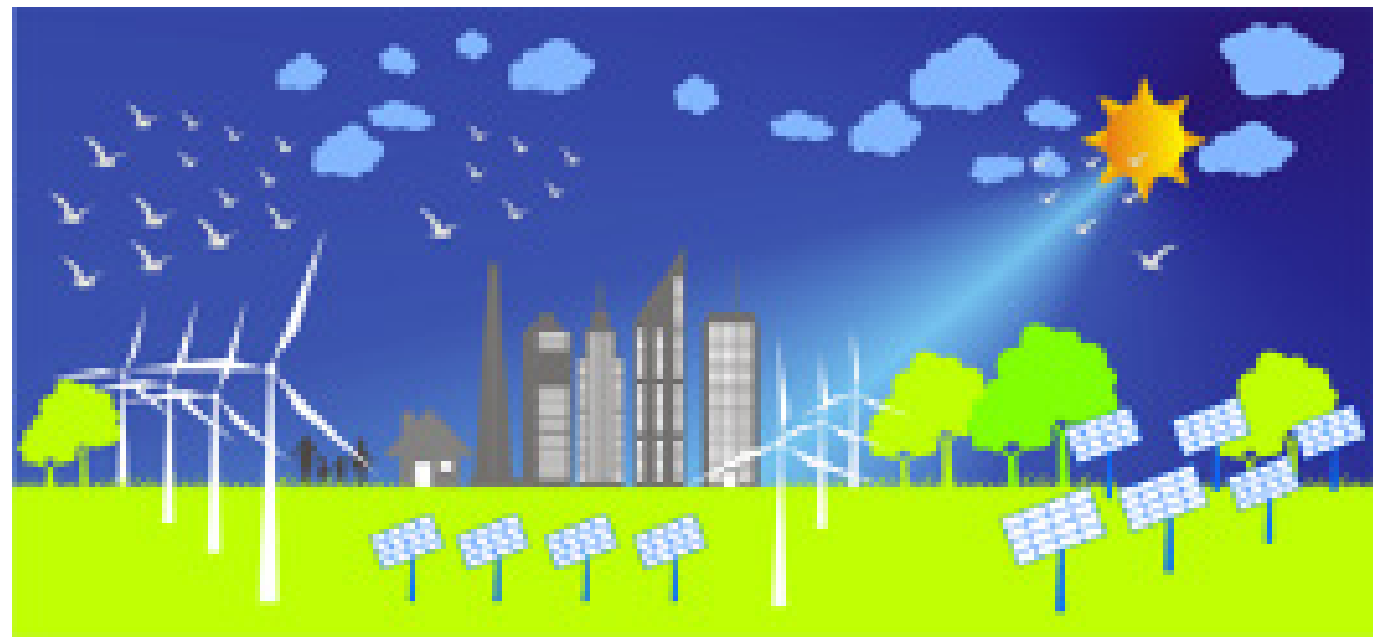

Esta transformación, en el mundo de las ideas y en las propuestas en medidas concretas, requerirá de una presentación del tema energético mediante un diálogo interdisciplinario entre las ciencias del medio ambiente y la ética ambiental. Cabe recordar que el avance científico-tecnológico es producto de una acción antropogénica, la humanidad no puede desprenderse del universo tecnológico ni del campo energético como motores de progreso y de calidad para alcanzar una vida ecológicamente sostenible y socialmente justa. Tras esto, subyace un saber ambiental que permita resignificar las reflexiones éticas hacia un futuro sustentable. 


\section{Conclusiones}

La acción antrópica, en actitud reconciliadora con el clima y la naturaleza, sigue siendo tan o más urgente. No caben dudas que se está en presencia de una clara crisis ecológica y civilizatoria que se despliegan a nivel mundial. De ahí surge la imperiosa celeridad de reconocer a la Naturaleza como sujeto y de generar relaciones armónicas entre la humanidad y la naturaleza. En consecuencia, es necesario lograr una reconstrucción del mundo en un lugar mejor, fundado en un saber ambiental con una concepción ética y bioética en el marco del paradigma de la vida, y que sitúe al hombre en una nueva era de conciencia biosférica.

La naturaleza inspira progreso e innovación: un progreso que va más allá de lo cuantitativo y que está orientado hacia la sustentabilidad ecológica; y una innovación tecnológica, que a la hora de diseñar e implementar modelos de desarrollo, respeta los límites homeostáticos de la esfera de la vida. Por suerte, en este punto crítico y apremiante para el futuro del planeta y de la humanidad, se juntan esfuerzos para encaminar a la sociedad hacia un desarrollo pleno de la integración de las energías renovables y hacia una eficiencia energética dentro del marco del desarrollo sostenible, ambos factores necesarios para la supervivencia planetaria.

Edificar sociedades sustentables, más justas e igualitarias, exige recurrir a energías amigables con el medioambiente. Se comienza a enarbolar banderas y a reivindicar los criterios éticos basados en el respeto, solidaridad e igualdad para una distribución más justa de los recursos naturales. Esta metamorfosis ideológica y paradigmática nos permite pensar en que es posible encontrar los medios necesarios para vivir de manera sostenible, caracterizados por el acceso equitativo a la salud, alimentación, vivienda, energía y educación. Actualmente, emerge un paradigma ambiental que propicia a un viraje radical en el modelo imperante, y a la formulación de una nueva visión ontológica en la estrecha interacción hombre-naturaleza.

\section{Referencias}

* Agoglia Moreno, O. B. (2010). La crisis ambiental como proceso. Un análisis reflexivo sobre su emergencia, desarrollo y profundización desde la perspectiva de la teoría crítica [Tesis doctoral, Universidad de Girona]. https://www.tesisenred.net/ bitstream/handle/10803/7671/tobam.pdf?sequence=

* Delgado Díaz, C. J. (2002). Cognición, problema ambiental y bioética. https:// www.academia.edu/43975605/Cognici\%C3\%B3n_problema_ambiental_y_ bio\%C3\%A9tica

- Kalmus, P. (2018). Cambio climático: la humanidad en la encrucijada. En F. González (Presidente, BBva), ¿Hacia una nueva Ilustración? Una década trascendente. (pp. 220-246). OpenMind BBVA. 
* Lacan, J. (2009). Escritos 1. Siglo xxı.

* Lecaros Urzúa, J. A. (2013). La ética medio ambiental: principios y valores para una ciudadanía responsable en la sociedad global. Acta Bioethica, 19(2), 177-188. http://dx.doi.org/10.4067/S1726-569X2013000200002

- Leff, E. (2007). Complejidad, racionalidad ambiental y diálogo de saberes: hacia una pedagogía ambiental. Desenvo/vimento e Meio Ambiente, (16), 11-19. http://doi. org/10.5380/dma.v16i0.11901

* Osorio García, S. N. (2016). Epistemología Axiológica y Bioética Global dos herramientas para la construcción de Proyectos Axiológicos Colectivos Pacs. Centro de Estudio de las Tradiciones de Sabiduría (CETR). https://cetr.net/epistemologiaaxiologica-y-bioetica-global-dos-herramientas-para-la-construccion-deproyectos-axiologicos-colectivos-pacs/

* Perino, E. J., Perino, E. y Perelló, A. D. (2019). Sustentabilidad: Un Nuevo Imperativo Categórico. Acta de la xLII Reunión de Trabajo de la Asociación Argentina de Energías Renovables y Medio Ambiente (ASADES), 7, 79-90.

Potter, V. R. (1971). Bioethics: Bridge to the future. Prenctice Hall.

* Rifkin, J. (2010, 19 de marzo). La civilización empática. El País. https://elpais.com/ diario/2010/03/19/opinion/1268953211_850215.html

* Sábato, E. (2000). La Resistencia. Planeta Argentina.

\section{Cómo CITAR ESTE ARTículo}

* Perino, Ernesto Jesús, Kiessling Duran, Roberto Aníbal, Silnik, Adolfo Alejandro, Perelló, Aníbal Daniel y Perino, Ernesto. (2021, mayo-junio). Energías renovables y sustentabilidad: una eficiente forma de gestionar los recursos naturales. Revista Digital Universitaria (RDU), 22(3). Dol: http://doi.org/10.22201/ cuaieed.16076079e.2021.22.3.4 